\section{PSQ-031 CLINICAL AND ECONOMIC IMPACT AFTER BREAKING THE SINGLE TABLET ABACAVIR/LAMIVUDINE/ DOLUTEGRAVIR COMBO TREATMENT INTO TWO DRUG REGIMENS}

P Perez Puente*, P Barriga Rodríguez, MR Garrido Ameigeiras, C Caba Hernández, P Tena Alejandre, JJ Duque Aguilar, J Sanchez Ferrer, S Arnaiz Diez, LC Fernández Lisón. Complejo Hospitalario Universitario De Caceres, Hospital Pharmacy, Caceres, Spain

10.1136/ejhpharm-2020-eahpconf.348

Background and importance In June 2018, our HIV regional working group, in a programme to improve the efficiency of antiretroviral therapy (ART), recommended changing from a single tablet regimen (STR) with abacavir/lamivudine/dolutegravir (ABC/3TC/DTG) once daily to abacavir/ lamivudine (ABC/3TC) generic plus dolutegravir (DTG) once daily.

Aim and objectives To evaluate the degree of implementation of this strategy and the impact in terms of adherence and efficiency after 9 months.

Material and methods The retrospective descriptive study (June 2018-March 2019) included all HIV patients treated with STR ABC/3TC/DTG. To measure adherence, the consumption and dispensation registry of the pharmacy service software programme was used. Patients with a value $>95 \%$ were considered adherent. The analytical variables collected were viral load (VL, copies/mL) and CD4 lymphocytes (cells $/ \mu \mathrm{L}$ ) (last available analytical before the change and at least 3 months later). Costs considered were hospital average prices according to the regional public tender.

Results Fifty-two patients, mean age 51.56 years, receiving treatment with $\mathrm{ABC} / 3 \mathrm{TC} / \mathrm{DTG}$, were included. The change in ART was carried out in all patients.

Forty-four patients (84.6\%) were adherent (>95\%) before the switch and remained so after the change. We detected 8 (15.4\%) patients with suboptimal adherence( $<95 \%$ ), with a mean adherence prior to the change of $81.5 \%$ (SD 5.3\%) and after the change 84.3\% (SD 6, 2\%). Before the change, 49 patients $(94.2 \%)$ presented undetectable VL, 2 patients (3.8\%) had between 50 and 200 copies/mL and 1 patient (1.9\%) had VL $>200$ copies/mL. After the change, 46 patients were evaluated (6 did not have analytics), 43 (93.4\%) with undetectable VL, 2 (4.3\%) with VL 50-200 copies/mL and 1 patient $(2.1 \%)$ with VL $>200$ copies $/ \mathrm{mL}$.

The average level of LCD4 in the pre-change analysis was $808.67 / \mu \mathrm{L}$ (SD 205) and after the switch $785.4 / \mu \mathrm{L}$ (SD 308).

Cost savings were $132 € /$ patient/month (1584€/patient/ year). The estimated savings for the hospital since this efficiency measure was implemented until March 2019 was 41 $000 €$.

Conclusion and relevance The results of the study, despite its limitations, demonstrated that after the switch, levels of virological suppression were maintained with a significant reduction in healthcare costs without affecting patient adherence to ART. More exhaustive and long term studies should be carried out to corroborate these results.

\section{REFERENCES AND/OR ACKNOWLEDGEMENTS}

No conflict of interest.

\section{PSQ-032 SUCCESSFUL TREATMENT OF CHRONIC HEPATITIS C INFECTION WITH CRUSHED SOFOSBUVIR/ VELPATASVIR}

${ }^{1}$ A Puebla Villaescusa*, ${ }^{1}$ I Gozalo Esteve, ${ }^{2} \mathrm{M}$ Larrea Vargas, ${ }^{1} \mathrm{MJ}$ Fraile Gallart. ${ }^{1}$ Hospital Sant Rafael, Pharmacy Service, Barcelona, Spain; ${ }^{2}$ Hospital Sant Rafael, Digestive Service, Barcelona, Spain

\subsection{6/ejhpharm-2020-eahpconf.349}

Background and importance Sofosbuvir/velpatasvir (SOF/VEL) is an oral regimen approved for patients with hepatitis $C$ virus (HCV). To date, no pharmacokinetic data exist on the impact on efficacy and safety of this regimen when it is crushed and mixed with liquids or food.

Aim and objectives To describe the case of a 65 -year-old man patient with HCV infection who successfully achieved a sustained viral response (SVR) when SOF/VEL oral combination was administered crushed and dissolved in $10 \mathrm{~mL}$ of water.

Material and methods A 65-year-old man presented with HCV infection, genotype 4, with minimal fibrosis (F0-F1). He was operated on for laryngeal neoplasia and treated with radiotherapy 10 years previously, presenting secondary swallowing problems since then. His last evaluation of liver fibrosis was $4.7 \mathrm{kPa}$ ( 1 year before treatment). He showed elevated levels of aspartate aminotransferase (43 U/L), alanine aminotransferase (48 U/L) and gamma-glutamyl transferase $(94 \mathrm{U} / \mathrm{L})$ at the beginning of treatment, and a normal range for other liver profile values. Off-label treatment with crushed SOF/VEL dissolved in $10 \mathrm{~mL}$ of water for 12 weeks was decided, and serum HCV-RNA was determined at +12 weeks, +24 weeks (SVR) and 1 year post-treatment.

Results The patient presented undetectable serum HCV-RNA at +12 weeks, +24 weeks (SVR) and 1 year post-treatment, and normal liver enzymes values were reached at +12 weeks post-treatment. SOF/VEL tablets only took $1 \mathrm{~min}$ to be dissolved in water, with a bitter taste, according to the patient. Conclusion and relevance Crushed SOF/VEL was effective in eradicating $\mathrm{HCV}$ in our patient. However, there is little evidence to support the practice of crushing SOF/VEL for reliable conclusions, and hence more studies are needed to determine its bioavailability when administrated in a way different from the conventional one. We aim to develop management guidelines for antiviral drugs with different administrations.

\section{REFERENCES AND/OR ACKNOWLEDGEMENTS}

No conflict of interest.

\section{PSQ-033 GLECAPREVIR/PIBRENTASVIR USE IN CHRONIC HEPATITIS C: EFFECTIVENESS AND SAFETY}

A Ruiz Gómez*, L Menéndez Naranjo, M Sáez Garrido, A Laorden Carrasco, M Díaz Ramón, JÁ Cano Molina, A Espuny Miró. Hospital Clínico Universitario Virgen De La Arrixaca, Pharmacy, Murcia, Spain

\subsection{6/ejhpharm-2020-eahpconf.350}

Background and importance Over the last few years there have been remarkable advances in chronic hepatitis $\mathrm{C}$ virus (HCV) drug development, and the goals of most new regimens have been increasing sustained viral response rates 
(SVR), improving tolerability and shortened treatment duration.

Aim and objectives To describe the use of glecaprevir/pibrentasvir in the treatment of $\mathrm{HCV}$ patients, as well as to evaluate efficacy and safety.

Material and methods This was an observational retrospective study in all adult HCV patients who received treatment with glecaprevir/pibrentasvir between December 2017 and December 2018. Variables collected were age, sex, genotype, degree of fibrosis, type of patient (naïve, relapsed or nonrespondent), prior treatment, treatment duration, basal viral load (VL), VL at 12 weeks after finishing treatment and adverse reactions. As an indicator of effectiveness, SVR was used.

Results A total of 37 patients $(70.27 \%$ men) were analysed with a median age of 54 years (range 20-81). Six patients (16.22\%) had genotype $1 \mathrm{a}, 10(27.03 \%)$ had genotype $1 \mathrm{~b}, 1$ $(2.70 \%)$ had genotype 2, $10(27.03 \%)$ had genotype 3 and $10(27.03 \%)$ had genotype 4 . Regarding the degree of fibrosis, 7 patients $(18.92 \%)$ were F0, $10(27.03 \%)$ were F1, 9 $(24.32 \%)$ were F2, 2 (5.41\%) were F3, 3 (8.11\%) were F4, and the degree of fibrosis was not determined in 6 patients $(16.22 \%)$. Thirty $(81.08 \%)$ were treatment naïve patients, 4 $(10.81 \%)$ failed prior treatment with interferon+ribavirin, 2 $(5.40 \%)$ were non-responders to treatment with direct acting antivirals (DAA) and $1(2.70 \%)$ was a non-responder to both interferon and DAA. Treatment duration was 8 weeks in 28 patients $(75.68 \%), 12$ weeks in $6(16.22 \%)$ and 16 weeks in 3 (8.11\%). Median baseline VL was $1506164 \mathrm{IU} / \mathrm{mL}$ (range 19 800-49 033 584), with 23 patients (62.16\%) having $>800000 \mathrm{IU} / \mathrm{mL}$. SVR was achieved in 33 patients (89.19\%). VL was not determined in three patients, although two of them presented undetectable VL at the end of treatment and one patient died before reaching 12 weeks post treatment. Regarding safety, six patients suffered at least one adverse reaction: nausea (2), fatigue (2), gastrointestinal discomfort (2), gas (1), night sweats (1), dry mouth (1), diarrhoea (1) and headache (1).

Conclusion and relevance Glecaprevir/pibrentasvir represents an effective pangenotypic therapeutic option for naïve, nonresponding and relapsing $\mathrm{HCV}$ patients due to the high percentage of patients who achieved SVR. Most of the adverse reactions reported were similar to those described in clinical trials, all of them being mild, and did not require interruption of treatment.

\section{REFERENCES AND/OR ACKNOWLEDGEMENTS}

No conflict of interest.

\section{PSQ-034 EVALUATION OF OSELTAMIVIR USE IN CLINICAL PRACTICE IN A SECOND LEVEL HOSPITAL}

${ }^{1} \mathrm{P}$ Taberner Bonastre*, 'Á Casinos Rodriguez, ' $\mathrm{L}$ Vállez Valero, 'M Cano Marrón, ${ }^{1} \mathrm{M}$ Martinez Sogues, ${ }^{1} \mathrm{~B}$ Martinez Castro, ${ }^{1} \mathrm{JA}$ Schoenenberger Arnaiz. 'Hospital Universitario Arnau De Vilanova, Hospital Pharmacy, Lleida, Spain; ${ }^{2}$ Hospital De La Santa Creu I Sant Pau, Hospital Pharmacy, Barcelona, Spain

\subsection{6/ejhpharm-2020-eahpconf.351}

Background and importance There is some controversy about the use of oseltamivir, dose adjustment and treatment duration. A new protocol was updated last year in our hospital specifying renal impairment posology adjustment criteria and cases in which the use of oseltamivir for $7-10$ days is justified: patients hospitalised in the intensive care unit (ICU), and patients receiving immunosuppressive or antineoplastics drugs.

Aim and objectives To evaluate the suitability of oseltamivir prescriptions based on the updated protocol in our hospital; to evaluate the pharmacist interventions related to oseltamivir prescriptions; and to analyse the simultaneous prescription of antibiotics in patients with influenza A.

Material and methods This was an observational retrospective study of adult patients with influenza A confirmed infection, treated with oseltamivir during the period December 2018 to February 2019. Paediatric patients and those hospitalised in the ICU were excluded. Demographic variables, unit of prescription, glomerular filtration rate (calculated by CDK-EPI), dosage, treatment duration and reasons to extend oseltamivir treatment were registered. Moreover, pharmaceutical recommendations related to prescription, concomitant use of antibiotics and the results of microbiological culture were gathered.

Results During the study period, 255 patients were included, $132(52.36 \%)$ men and $176(68.12 \%)$ aged >65 years (20-98 years). The units of prescription were: surgical $6.3 \%$ and medical $93.7 \%$. Posology was not suitable to renal impairment in 17 cases (6.7\%). A total of 42 patients received oseltamivir for a period of time other than 5 days: in 36 patients $(85.7 \%)$ the reasons were not justified and in 6 patients $(14.3 \%)$ were due to ICU admission and use of immunosuppressive drugs. Eighty-two pharmaceutical interventions were done: $17(20.7 \%)$ related to posology of which 58.8\% were accepted and 65 (79.3) related to the duration of oseltamivir of which $90.8 \%$ were accepted. Of all the patients included, 119 (46.9\%) were also prescribed an antibiotic, in 31 of whom a microorganism was isolated.

Conclusion and relevance The degree of compliance with the oseltamivir hospital protocol updated in 2018 was $>80 \%$. In total, $>90 \%$ of the pharmaceutical interventions were accepted resulting in a change in the medical prescription according to the protocol recommendations. Pharmaceutical validation adds safety to the hospitalised patient and optimised oseltamivir prescription.

\section{REFERENCES AND/OR ACKNOWLEDGEMENTS}

No conflict of interest.

\section{PSQ-035 DUAL THERAPY WITH DOLUTEGRAVIR AND LAMIVUDINE: EFFICACY AND SAFETY}

F Toja*, M Pereira Vazquez. Complejo Hospitalario Ourense, Pharmacy Service, Ourense, Spain

\subsection{6/ejhpharm-2020-eahpconf.352}

Background and importance A non-comparative study, a randomised pilot clinical trial and a cohort suggest that the change is virologically safe. There are still no results from two large randomised clinical trials in development. In naive patients, this pattern has shown no inferiority of dolutegravir and lamivudine compared with triple treatment with dolutegravir plus tenofovir/emtricitabine.

Aim and objectives To evaluate the efficacy, economic impact and reduction in adverse effects in HIV patients undergoing bi-therapy treatment with dolutegravir and lamivudine.

Material and methods A retrospective observational study was conducted in a second level hospital. Patients who started 